\title{
10 Diskussion der Ergebnisse
}

Im Folgenden werden die Ergebnisse dieser Studie entlang von vier Aspekten diskutiert: Zunächst wird die Wahl des analytischen und methodologischen Frameworks reflektiert sowie das Verhältnis zwischen Mikro- und Makro-Ebene in den Blick genommen (Abschnitt 10.1). In Abschnitt 10.2 werden Serien mit anderen medialen Gesprächsgegenständen verglichen und die sich daraus ergebenden Implikationen diskutiert. Anschließend werden die spezifischen Anforderungen, die Serienkommunikation an das interaktive Management von Wissen und Bewertungen stellt, erläutert (Abschnitt 10.3). Schließlich stehen in Abschnitt 10.4 die Akteur^innen selbst im Fokus und es wird diskutiert, inwiefern sie sich als kompetente Serienrezipient*innen positionieren, bevor in Abschnitt 10.5 eine Zusammenfassung und ein Ausblick erfolgen.

\subsection{Die Analyse von Medienkommunikation und das Mikro-Makro-Problem}

Für einen umfassenden analytischen Zugriff auf Serienkommunikation als Form von Medienkommunikation wird zunächst grundsätzlich dafür plädiert, sowohl mikroanalytisch als auch makroanalytisch vorzugehen. Für die mikroanalytische Perspektive bietet die Konversationsanalyse (Abschnitt 4.1) einen sinnvollen Zugang an. Mit ihren Maximen, offen und datengeleitet Interaktionen aus der Teilnehmendenperspektive heraus zu untersuchen, können der sequenzielle Kontext angemessen berücksichtigt sowie die spezifischen interaktiven Ziele und kommunikativen Aufgaben Interagierender in Gesprächssituationen rekonstruiert werden. Auch abseits der Analyse eines elizitierten Interaktionssettings erscheint eine solche Vorgehensweise zielführend. So wird bei jeder Form der Datenerhebung, die nicht verdeckt stattfindet, eine Form von Öffentlichkeit hergestellt (Abschnitt 6.1.2), die reflexionsbedürftig ist. Auch kann es sinnvoll sein, Kontextualisierungshinweise bezüglich der Herstellung von Institutionalität zu berücksichtigen, da die Grenze zwischen alltäglicher und institutionalisierter Interaktion empirisch nicht immer eindeutig bestimmbar ist (vgl. Meer 2011).

Ein Erkenntnisinteresse konversationsanalytisch basierter Datenanalyse besteht darin, regelhaft organisierte interaktive Praktiken identifizieren zu können, die einerseits kontextspezifisch sind, andererseits für die rekonstruierten Funktionen aber auch wieder kontextübergreifend typisch sind (vgl. auch Deppermann, Feilke und Linke 2016). So lassen sich die für Serienkommunikation typischen Praktiken entlang der epistemischen Positionierung der Interagierenden systemati- 
sieren (Abschnitte 7.1 und 7.2). Über die kontextuelle Einbettung kann aber zugleich auch analysiert werden, ob Praktiken beispielsweise als Scherzinteraktion gerahmt sind (Abschnitt 6.5.2.3) oder ob Inszenierungen vor und für andere Interagierende, die Kamera und/oder Forschende als Interpretationsressource einbezogen werden müssen (Abschnitt 6.1.2). Dadurch kann auch herausgearbeitet werden, ob in einer Sequenz eher eine Präferenz für Konsens (Abschnitt 7.3.2) oder Dissens (Abschnitt 7.3.3) vorherrscht sowie ob für die Beteiligten das Bearbeiten von Wissensasymmetrien (Abschnitt 7.1.1) im Vordergrund steht oder eher face-work betrieben wird (Abschnitt 9.1.1).

In Kombination mit Positionierungsanalyse kann nun nicht nur nachgezeichnet werden, wie diese Praktiken in dem gegenwärtigen Gesprächskontext situiert sind, sondern darüber hinaus können auch implizite Verweise auf gegenstandsbezogene Diskurse sichtbar gemacht werden. Das Sprechen über die eigene emotionale Beteiligung bei der Rezeption (Abschnitt 8.2.4.1) oder die als angemessen behandelte Einteilung zeitlicher Ressourcen (Abschnitt 8.2.4.3) sind zwar - unter anderem - an Serien als Gesprächsgegenstand geknüpft, werden aber jeweils kontextuell unterschiedlich realisiert. So zeigt etwa die Analyse der Interaktionssequenzen zwischen zwei Teilnehmenden aus einer Gruppe im Gesprächsverlauf, dass die Positionierungspraktiken, die sie jeweils anwenden (Absprechen fremder epistemischer Autorität, Anzeigen partiellen Wissens), einerseits kontextsensitiv in eine vergnügliche Dissens-Sequenz eingebunden sind. Andererseits lässt sich über diese Praktiken auch rekonstruieren, dass beide in unterschiedlicher Art und Weise auf übergeordnete, prototypische bzw. normative Vorstellungen serieller Figuren verweisen (vgl. Abschnitte 7.1.3 und 9.1.1). Wie in Kapitel 9 demonstriert wurde, können so mikro- und makroanalytische Befunde systematisch aufeinander bezogen werden, um dynamische Vergemeinschaftungs- und Distinktionsaktivitäten beschreiben und interpretieren zu können.

Der Mehrwert der Verknüpfung beider Ansätze liegt also darin, dem indexikalischen Charakter von Kommunikation gerecht zu werden und sowohl den sequenziellen Kontext als auch größere Diskurse für die Untersuchung von Vergemeinschaftung und Distinktion einzubeziehen. Um Positionierungsanalyse mit Konversationsanalyse zu kombinieren, hat es sich als sinnvoll erwiesen, das Verständnis von Positionierung, wie es in Abschnitt 3.3 (Merkmale (1) bis (7)) beschrieben wird, zugrunde zu legen. Positionierungen werden in diesem Sinne als ein interaktiv hergestelltes Phänomen modelliert, das (1) kontextgebunden, (2) interaktiv und relational, (3) dynamisch, (4) gegenstandsbezogen, (5) potentiell ubiquitär, (6) explizit und implizit bzw. von unterschiedlicher Intensität sowie (7) räumlich im wortwörtlichen und/oder metaphorischen Sinne ist. Die zur Erfassung der Komplexität von Medien- und Rezeptionskommunikation erforderliche holistische Analyseperspektive bildet das erarbeitete Modell visuell ab (vgl. Abbil- 
dung 9.1), indem es veranschaulicht, wie die verschiedenen Analysedimensionen zusammenhängen. Mit dem Modell wurde ein Beschreibungsinstrumentarium mit heuristischer Charakteristik entwickelt, um Vergemeinschaftungs- und Distinktionsaktivitäten als eine der Kernfunktionen von Medienkommunikation (vgl. Abschnitt 2.1.3 und 2.2.3) analytisch erfassen zu können. Die Reichweite der so generierten Befunde über die in dieser Arbeit in den Blick genommenen Daten hinaus ließe sich in weiteren Studien untersuchen.

\subsection{Serienkommunikation im Vergleich zu anderen medialen Gesprächsgegenständen}

In Abschnitt 2.2.2 wurde diese Studie bereits in den Kontext von Kommunikation über Fernsehen, Literatur, Kunst und Theater gestellt sowie wichtige Erkenntnisse dieser Forschungsergebnisse für die Analyse nutzbar gemacht (Abschnitt 2.2.3). Im Folgenden werden die Ergebnisse der Studie mit anderen Formen der Medienkommunikation verglichen. Die Diskussion erfolgt entlang des Vergleichs der (strukturellen) Besonderheiten der Kommunikation, die sich sowohl in gesprächskontextuellen Aspekten als auch in den Affordanzen der Medienprodukte und ihrer Rezeption niederschlagen.

Zunächst lässt sich konstatieren, dass es sich bei der untersuchten Kommunikationsform nicht um rezeptionsbegleitende Interaktion handelt. Das ist insofern relevant, als die geteilte Wahrnehmung eines Objekts (als ,joint attention“, vgl. Tomasello und Farrar 1986) Auswirkungen auf die jeweiligen diskursiven Anforderungen hat. So sind für Aussagen über das Medienprodukt unterschiedlich aufwändige rekonstruktive Verfahren sowie verschiedene Formen der Deixis erforderlich, abhängig davon, ob der mediale Gegenstand in der Interaktionssituation selbst - in einer Kunstausstellung, gemeinsam vor einem Endgerät usw. oder kurz danach - in der Theaterpause, direkt nach dem gemeinsamen Kinobesuch usw. - für alle Beteiligten wahrnehmbar ist. Vor dem Hintergrund des Situationsarrangements der Studie (vgl. dazu Abschnitt 5.1.1) ist eine gemeinsame Rezeptionshistorie der Teilnehmenden eher unwahrscheinlich. ${ }^{1}$ Stattdessen erfordert es die forscherinnenseitige Instruktion von den Teilnehmenden (Abschnitt 6.1.1), nahezu ausschließlich Medienrekonstruktionen (Abschnitt 6.5.2.3; vgl. auch Keppler 1994) zu produzieren, um die kommunikativen Aufgaben zu bearbeiten. Inwiefern dieser Aspekt auch in nicht-elizitierten Settings ebenfalls eine Rolle

1 Eine Ausnahme bildet die gemeinsame Rezeption von CRIMINAL Minds von Emilia und Martha, vgl. Abschnitt 8.2.4.1. 
spielt, bleibt empirisch zu klären. Ausgehend von dem dieser Studie zugrunde gelegten Praktikenbegriff (Abschnitt 4.1) liegt jedoch die Vermutung nahe, dass die vorliegende Studie zwar eine spezifische Variante von Serienkommunikation erfasst, die identifizierten Merkmale, Formen und Funktionen sich aber auch auf informellere Kontexte übertragen lassen.

Den meisten Studienergebnissen zufolge (vgl. Abschnitt 2.2.3) ist dem Austausch über mediale Produkte gemeinsam, dass diese Gespräche sowohl Bewertungs- als auch Wissens-, Scherz- sowie Bedeutungskommunikation in unterschiedlichem Maße erwartbar machen. Im Rahmen der vorliegenden Untersuchung wurde gezeigt, dass in Gesprächen über Serien Bewertungen und Wissensinteraktionen omnipräsent sind und beidem ein zentraler Stellenwert zukommt (vgl. Abschnitt 6.3). Auch wenn die Teilnehmenden durchaus Vergnügen am Dissens signalisieren und ihre Interaktion scherzhaft rahmen bzw. Frotzelaktivitäten integrieren (vgl. z.B. Analysen in Abschnitt 7.1.3), lässt sich jedoch daran keine durchgängige Orientierung erkennen (vgl. etwa die Analysen in Abschnitt 8.2.4.1). Gleichermaßen handeln die Jugendlichen zwar bisweilen Bedeutungen und Interpretationsansätze aus (vgl. Analysen in Abschnitt 7.1.4), bleiben aber meistens eher auf der deskriptiven Handlungsebene. Dabei konnte mit Hilfe des Modells gezeigt werden, dass die jeweiligen Kommunikationsformen für kontextuell angemessene Vergemeinschaftungs- bzw. Distinktionsaktivitäten funktionalisiert werden können.

Werden diese Befunde mit den Ergebnissen anderer Forschenden verglichen, zeigen sich folgende Unterschiede: Androutsopoulos und Weidenhöfer (2015: 34) nehmen in ihrer Ergänzung zu den Kategorien fernsehbegleitenden Sprechens nach Klemm (2000) an, dass zusätzlich zum Bewerten - anders als in dieser Studie - das Sich vergnügen eine übergeordnete Handlungskategorie von Rezeptionskommunikation darstellt. Das ist vermutlich auf die zwar räumliche, nicht aber zeitliche Entkopplung von Rezeptions- und Kommunikationssituation ihres Forschungsgegenstands (Twitter) zurückzuführen. Grundsätzlich scheint also unter anderem das Interaktionssetting einen Einfluss auf die Wahrscheinlichkeit des Zustandekommens der verschieden Handlungskategorien zu haben. So stellen Charlton und Sutter (2007: 142) im Rahmen des Vergleichs ihrer Ergebnisse mit Hausendorfs Untersuchungen fest, dass Bewertungen als „der eigentliche Fluchtpunkt der Kunstkommunikation“ (Hausendorf 2007: 35) in ihren Daten kaum eine Rolle spielen. Diese Beobachtung führen sie auf die institutionelle Rahmung der Situation zurück, d.h. dass die beforschten Schülerinnen primär die Erwartungen des Literaturunterrichts bedienen und daher - im Unterschied zu dieser Studie - vorrangig Deutungen und Interpretationen statt (subjektiver) Bewertungen produzieren. Die Relevanz der kontextuellen Einbindung der Gespräche in eine Erhebungssituation - sei sie elizitiert oder nicht - kann daher im Rahmen der vorliegenden Studie 
bestätigt werden. Weitere Erklärungen für Unterschiede zwischen den verschiedenen Formen der Medienkommunikation mögen in der zeitlichen Distanz zwischen Rezeptions- und Kommunikationssituation (s.o.), der diskursiven Verhandlung des Gegenstands in konkreter oder abstrakter Form (Abschnitt 6.5.1, vgl. weiterführend 10.3) sowie in den unterschiedlichen Affordanzen , die das Medium bereitstellt, begründet sein. Letzteres bedeutet, dass die verschiedenen Beschaffenheiten der ästhetischen Erzeugnisse unterschiedliche semiotische Kanäle bedienen und dass sie einen unterschiedlich hohen Grad an Mehrdeutigkeit und bereitgestellten sowie diskursiv zugeschriebenen Bedeutungen und Wertungen aufweisen. Dadurch verändern sich auch die Bedingungen ihrer Rezeption (vgl. Abschnitt 2.2.1), woraus sich wiederum Auswirkungen auf die Kommunikation ergeben können. Im Folgenden werden diese Aspekte diskutiert.

Audiovisuelle Serien, wie sie in dieser Studie als Gesprächsgegenstand vorgegeben wurden, weisen überwiegend einen weniger hohen Grad an Mehrdeutigkeit auf als beispielsweise Lyrik oder Kunstwerke. Dennoch können selbstverständlich ihre eingeschriebenen sowie diesbezüglich subjektiv konstruierten Bedeutungen ausgehandelt werden (vgl. Analysen in den Abschnitten 7.1.4 und 8.2.1). Der Grad der Mehrdeutigkeit hängt auch von der Komplexität der jeweiligen Serie ab (Abschnitt 8.1.3). Insbesondere über die zunehmende narratologische Komplexität wurde versucht, Serien nicht mehr unter „Trivialitätsverdacht“ (Anders und Staiger 2016: 9) zu stellen, sondern ihnen eine hochkulturelle Bedeutung zukommen zu lassen. Bei der diskursiven Zuordnung einzelner Serien oder Serien an sich zur Populär- oder Hochkultur handelt es sich jedoch um einen dynamischen Prozess, der sozialen und kulturellen Bedeutungszuweisungen unterliegt. So wurde bereits anhand des Exkurses zum Lesesucht-Diskurs in Abschnitt 2.1.3 deutlich, dass auch Gespräche über Literatur einem Wandel in der gesellschaftlichen Wahrnehmung unterliegen und nicht ohne Bezug dazu analysiert werden können (vgl. auch die Darstellung und Diskussion der Ergebnisse von Wiesners (2014) Studie in Abschnitt 3.2.5). Die Analyse der vorliegenden Daten zeigt, dass sich die Teilnehmenden an solchen Diskursen implizit orientieren (Kapitel 8), insbesondere an dem Sucht(Abschnitt 8.2.4.2) und Zeit- bzw. Intensitätsaspekt (Abschnitte 8.2.4.3 und 8.2.4.2), was sich sprachlich-interaktiv etwa in Rechtfertigungsaktivitäten für vermeintlich triviale und weniger komplexe Formate (vgl. v.a. die Analysen in Abschnitt 7.3.3, 8.1.3 und 8.2.2) widerspiegelt.

Weiterhin zählt zu den Charakteristika von Serien, dass ihre Produktion, Präsentation und Rezeption vermehrte Individualisierungs- und Optionalisierungstendenzen aufweist (vgl. Abschnitt 2.1.2). Serien - bzw. einzelne Episoden, Staffeln und Szenen - können über online-basierte Angebote theoretisch unendlich oft wie- 
derholt, pausiert oder übersprungen werden. ${ }^{2}$ Auch andere Formen medialer und literarischer Rezeption verändern sich unter den Bedingungen der Digitalität (vgl. diesbezüglich beispielsweise zum Lesen Krommer und Wampfler 2019). Allerdings bleiben spezifische Unterschiede bestehen. So können beispielsweise Kunstwerke im Rahmen einer Ausstellung zwar theoretisch unbegrenzt lange betrachtet werden; wenn die Rezipientin allerdings außerhalb des Ausstellungsraums keinen Zugang zu den Werken hat, ist ihr eine wiederholte Rezeption nur eingeschränkt möglich. Anders verhält es sich bei Theateraufführungen, die aufgrund ihres performativen Charakters notwendigerweise linear und - sofern sie nicht medial aufgezeichnet und gespeichert wurden - nicht sofort bzw. jederzeit wiederholbar sind. In Ansätzen konnte im Rahmen der vorliegenden Studie gezeigt werden, dass sich die auflösenden Verhältnisse der Linearität von Serienrezeption auf die sprachliche Oberfläche der Rezeptionskommunikation auswirken, wie etwa die Änderungen von Tempus und Modus einer Teilnehmerin im Verlauf einer Sequenz zeigen (vgl. Abschnitt 7.3.2). Allerdings handelt es sich dabei um einen explorativen und mit Vorsicht zu interpretierenden Befund, der keine generalisierenden Aussagen erlaubt.

Insgesamt haben Rezipient*innen also im Zuge der digitalen Transformation diverse Steuerungs- und Wahlmöglichkeiten bezüglich der Rezeption einer Serie, die sie als niedrigschwellig und daher positiv behandeln (vgl. Analysen in Abschnitt 8.2.4.4). Diese Individualisierungstendenzen führen im Kontext von Serienrezeption dazu, zunächst einen common ground etablieren zu müssen, bevor tiefere Deutungen und Interpretationen konkreter Serien diskursiv erörtert werden (können). Dieser Punkt wird im folgenden Abschnitt weiterführend diskutiert.

\subsection{Epistemische Besonderheiten der Serienkommunikation}

Der deutsche Terminus der Anschlusskommunikation hat sich für Gesprächsformen etabliert, die nach der Rezeption eines medialen Gegenstands entstehen. Hier kann kritisch angemerkt werden, dass diese Definition aus den beiden folgenden Gründen als nicht ganz trennscharf angenommen werden kann: Zum einen können beispielsweise literarische Gespräche, die oft auch als Anschlusskommunikation bezeichnet werden - vgl. für lese- und literaturdidaktische Konzeptionen z.B. die soziale Ebene in dem Modell nach Rosebrock und Nix (2014: 112) ${ }^{3}$ - , ebenfalls

2 Vergleichbar mit den Möglichkeiten, die Videoaufnahmen bezüglich der Verarbeitung und Aufbereitung von Daten zur Transkription ermöglicht haben (vgl. Abschnitt 4.1).

3 Vgl. zur Kritik an Anschlusskommunikation als „unschöne[m] Begriff“ (Spinner 2006: 12). 
rezeptionsbegleitend sein; nämlich dann, wenn Interagierende während des Gesprächs visuellen Zugriff auf den literarischen Text haben (vgl. auch Ausführungen in Abschnitt 10.2). Daher kann zum anderen davon ausgegangen werden, dass sowohl bei rezeptionsbegleitender Kommunikation als auch bei Anschlusskommunikation üblicherweise alle Beteiligten einander zuschreiben, epistemischen Zugang zu dem Gegenstand zu haben, also dasselbe Kunstwerk oder Bilderbuch $\mathrm{zu}$ betrachten oder betrachtet $\mathrm{zu}$ haben, dieselbe Theateraufführung zu sehen oder gesehen zu haben, dasselbe Gedicht zu lesen oder gelesen zu haben usw. Allerdings sind Interagierende dabei lediglich auf Beobachtungen, Erfahrungen und darauf basierende Schlussfolgerungen angewiesen. Sie können weder wissen, ob die anderen Personen das Medienprodukt tatsächlich rezipiert haben, noch ob sie es in ähnlicher Weise wahrgenommen haben, da Rezeption ein aktiver und individueller Bedeutungskonstruktionsprozess ist (vgl. Abschnitt 2.2.1).

Insbesondere letzterer Punkt ist charakteristisch für Kommunikation über Medien als ästhetisch gestaltete Erzeugnisse, die unterschiedliche Grade von Mehrdeutigkeit zulassen. Das wird unter anderem daran deutlich, dass Wissenskommunikation auch bei der gemeinsamen Rezeption und gleichzeitigen Wahrnehmung eines Medienprodukts salient wird, wie etwa die informierenden Rekonstruktionen in Foyergesprächen (Schlinkmann 2018) oder die Handlungskategorien des Informierens und Verständnissicherns bei der fernsehbegleitenden Kommunikation (Klemm 2000) zeigen. Dieser Aspekt wird wiederum in Bildungskontexten oft gezielt genutzt, um Lernprozesse anzuregen (vgl. etwa das Ziel literarischen Lernens über das literarische Gespräch (Spinner 2006: 12) oder Studien zum Lernertrag bei Kindern aus Vorlesegesprächen (vgl. z.B. Preußer 2017 und Wieler 2008)).

Werden diese Überlegungen mit den in Abschnitt 4.3 skizzierten Modellierungen von Wissensdomänen zusammengebracht, so können Medienprodukte als potentielle AB-Events (Labov und Fanshel 1977) verstanden werden, zu denen zumindest theoretisch - alle Interagierenden epistemischen Zugang haben können. Dazu zählen insbesondere jene Serien, die nicht über lineares Fernsehen ausgestrahlt werden, sondern digital verfügbar sind - wenn auch ggf. mit Kosten verbunden - und damit jederzeit zur Rezeption bereit stehen. Die eigentliche Rezeption und damit die subjektive Erfahrung eines medialen Erzeugnisses unterscheidet sich jedoch von Individuum zu Individuum und kann daher als A-Event bzw. type-1 knowable (Pomerantz 1980) bezeichnet werden, also als etwas, zu dem nur die oder der Rezipient`in selbst Zugang hat. Dennoch ist es möglich, solche Hypothesen und Annahmen über Rezeptionsverhalten und -erfahrungen anderer Personen zu treffen, die auf als geteilt behandelten, oft normativen Vorstellungen (vgl. Abschnitt 8.2) basieren, wie z.B., dass manche Serien einen emotionaleren Rezeptionsmodus nahelegen (vgl. Abschnitt 8.2.4.1) oder eine nachvollziehbare Handlungslogik aufweisen sollten (vgl. Abschnitt 8.2.1). Dadurch kann auch 
eigentlich rein subjektives und individuelles Erleben wie eine in hohem Maße identifikatorische Rezeption als kollektive Erfahrung, vergleichbar mit dem Erinnern an gemeinsame Erlebnisse (Bietti 2011), markiert werden (vgl. die Analysen in Abschnitt 7.3.1). Da Interagierende - im Rahmen der vorliegenden Daten auch bedingt durch das Studiendesign (Abschnitt 5.1.1) - nicht immer davon ausgehen können, dass allen Beteiligten jede Serie gleichermaßen bekannt ist, stellt das Etablieren eines common grounds eine priorisierte kommunikative Aufgabe im Kontext von Serienkommunikation dar (vgl. Abschnitt 6.3.2).

Aus diesem Grund wurde in das Analysemodell die Unterscheidung zwischen konkreten und abstrakten Positionierungsobjekten integriert (Abschnitt 6.5.1). Diese Unterscheidung erweist sich aus empirischer Perspektive als gewinnbringend, da sich in den vorliegenden Daten zeigt, dass die Teilnehmenden kontinuierlich den Fokus zwischen konkret und abstrakt verschieben. So greifen sie etwa bei der Diskussion über konkrete Serien auf abstraktes Wissen zurück (Abschnitt 7.1.2) oder nutzen konkrete Serien als Belege für abstraktere Behauptungen oder Thesen (Abschnitt 8.3). Es erscheint naheliegend, dass sich diese Unterscheidung auf andere mediale Formen ebenfalls übertragen lässt: Wer häufige ${ }^{\star}{ }^{*}$ Theatergänger`in ist, mag zwar ggf. nicht die in einem Gespräch thematisierte aktuelle Aufführung eines Stücks kennen, sich aber dennoch mit theaterbezogenem Wissen und Bewertungen, die sich aus anderen (Rezeptions-)erfahrungen speisen, auf abstrakter Ebene beteiligen. Gesprächsorganisatorisch kann gezeigt werden, dass sich die Anforderungen zur Partizipation an Diskussionen über konkrete oder abstrakte Objekte unterscheiden. Um über konkrete Serien zu sprechen - und insbesondere das epistemische Recht auf Evaluation zu beanspruchen und zu verteidigen -, setzen die Teilnehmenden untereinander einen minimalen epistemischen Zugang dazu voraus (vgl. Praktiken in Abschnitt 7.1 und 7.2; insbesondere Abschnitt 7.1.3).

In diesem Zuge bietet die Praktik des Managens divergenter Wissens(be)stände zwar einerseits eine Möglichkeit, mit dem variablen common ground der Serienkommunikation diskursiv umzugehen, sie bildet zugleich aber auch eine Herausforderung für die Interagierenden. So müssen sie zu Zwecken der Partizipation Wissensasymmetrien ausgleichen, dürfen allerdings nicht zu viele Informationen angeben, da sie sonst gegen die implizite Norm, nicht zu spoilern, verstoßen würden (Abschnitt 8.2.3). Daher bleiben Medienrekonstruktionen bei unterschiedlichen epistemischen Status der Interagierenden oft auch eher oberflächlich bzw. beziehen sich auf wenige Handlungselemente, gehen weder allzu sehr in die Tiefe noch transportieren sie verschiedene Deutungsansätze (vgl. Abschnitt 10.2). Die Anforderungen an den Wissenstransfer in diesem Kontext gleichen gewissermaßen einem Trailer oder einem Klappentext: Zwar müssen die wichtigsten Informationen vermittelt werden, um andere zum Rezipieren zu animieren, es dürfen aber nicht zu viele Informationen preisgegeben werden, um Spannung 
und Neugier aufrechtzuerhalten. Beim Sprechen über konkrete Serien - bzw. über spezifische, unterhaltende Medienprodukte - lässt sich also eine abweichende Präferenzstruktur bezüglich des Ausgleichs von Wissensasymmetrien erkennen.

Andererseits zeigen die Analysen aber auch, dass bei einem $\mathrm{K}^{-}$-Status in Bezug auf eine Serie Partizipation gleichermaßen durch Verweise auf abstrakte Aspekte gelingen kann (vgl. auch die Darstellung der Praktiken in Abschnitt 7.2). Dafür ist ebenfalls ein gewisses Maß an Wissen über diese Art von Gesprächen notwendig (vgl. dazu auch Habscheid 2018: 183), vergleichbar mit der Teilnahme an literarischen Gesprächen, aber auch mit dem Sprechen „über Bücher [...], die man nicht gelesen hat“ (Bayard 2007). Es lässt sich jedoch annehmen, dass das - demonstrierte - prototypische Wissen in Bezug auf abstrakte serienbezogene Aspekte (Abschnitt 8.1) umso höher ist, je mehr konkrete Serien die Teilnehmenden kennen. Der Erwerb prototypischen Wissens über serielle Formate ist dennoch keinesfalls auf audiovisuelle Serien beschränkt. Vielmehr sind viele erzählende Medienformate seriell strukturiert (vgl. auch Ausführungen in Abschnitt 2.1), so dass implizites Wissen über Serien auch durch Hörspielreihen, Comics, Bilderbücher, Romane usw. erworben werden kann. Darüber argumentieren auch Anders und Staiger, wenn sie im Kontext des Literaturunterrichts davon ausgehen, dass Schüler^innen bereits „Serienprofis“ (2016: 9) seien (vgl. Kapitel 1). Im Folgenden wird vor diesem Hintergrund der Fokus auf die kommunizierenden Rezipient`innen gelegt.

\subsection{Doing being kompetente Serienrezipient*innen}

Die in Abschnitt 10.3 erläuterte Relevanz der Unterscheidung zwischen konkretem und abstraktem Sprechen über Serien spielt auch mit Blick auf die Interagierenden und dementsprechend auf die Rezipient`innen eine Rolle. Gothe und Leichner bezeichnen Personen, die „sich für Serien ,an sich“ bzw. für das Format der Serie begeistern [...] [als] Serialisten“ (Gothe und Leichner 2012173; vgl. Abschnitt 2.1.2). Ähnlich bezieht sich Hausendorf mit den sozialen Kategorien „Kunstkenner“ und „Kunstsammler“ im Unterschied zu „Kunstbetrachtern“ auf Personen, die sich im Kunstsystem so positionieren, dass sie nicht nur ästhetischen Gefallen an einzelnen Werken signalisieren, sondern zeigen, dass sie Kunst an sich schätzen und sich damit auskennen (vgl. Abschnitt 2.2.2). Weder der Begriff der Serialistinnen noch der der Serienkenner ${ }^{\star}$ in bzw. -betrachter ${ }^{\star}$ in erweist sich allerdings für die vorliegende Studie als zielführend. Denn im Erkenntnisinteresse steht weniger die Einbettung einer sozialen Position in ein institutionelles Feld, sondern die Betrachtung des Zusammenspiels der kommunizierten Begeisterung für Serien, also die evaluative Dimension von Positionierungen, auf der einen Seite mit der episte- 
mischen Dimension, also der relativen Positionierung als Expert*in für Serien auf der anderen Seite.

Das ergänzend zum Analysemodell entwickelte Koordinatensystem (Abbildung 9.2) könnte den voreiligen Schluss nahelegen, alle Personen, die sich als wissende Unterstützer*innen oder Kritiker*innen einer Serie positionieren, als Serienprofis einzuordnen. Dieser Schluss greift jedoch zu kurz, denn die Analysen zeigen, dass die Teilnehmenden auch bei einem kommunizierten $K^{-}$-Status bezüglich einer Serie Praktiken nutzen, die signalisieren, dass sie sich zwar nicht mit dieser Serie, wohl aber mit Serien und Serialität auskennen und in diesem Zusammenhang auf Bekanntes, z.B. Genre-Wissen (Abschnitt 8.1.2) oder Wissen über Figurenkonstellationen (Abschnitt 8.1.4), zurückgreifen (können). Mit Hilfe des zu dem erarbeiteten Modell zählenden Koordinatensystems können Individuen vor dem Hintergrund dieser Befunde eingeordnet und deren relative epistemische und evaluative Positionierung in Bezug auf verschiedene Serien im Laufe eines Gesprächs miteinander verglichen werden.

Wird davon ausgegangen, dass bei Serienkommunikation Identitätsfacetten relevant werden, die sich auf Expertise und Geschmack beziehen (Abschnitt 3.1), so lässt sich festhalten, dass es für das Management dieser Identitätsfacetten notwendig ist, beides unter Einbezug der kommunikativen Anforderungen zu signalisieren. Interagierende, die Expertise zeigen, managen verschiedene Wissens(be)stände (Abschnitt 7.1.1) und stufen je nach den sequenziell folgenden Positionen der anderen Beteiligten ihre Glaubwürdigkeit oder Gewissheit mit weiteren Praktiken (Abschnitte 7.1.2 bis 7.1.4 und 7.2.1 bis 7.2.4) hoch. Dabei berücksichtigen sie die speziellen Anforderungen der Wissenskommunikation im Kontext von Seriengesprächen (Abschnitt 10.3) und platzieren wissenskommunikative Äußerungen kontextuell passend (vgl. die jeweils für die Praktiken herausgearbeiteten Strukturstellen in den Abschnitten 7.1 und 7.2). Die Wissensquelle ist dabei entscheidend, denn dem Primat der eigenen Erfahrung als knowable type-1 kann auch eine glaubwürdige andere Autorität gegenüberstehen (Abschnitt 7.1.2). Für das Anzeigen von allgemein serienbezogener Expertise ist aber vor allem die Kenntnis prototypischer Muster (Abschnitt 8.1) konstitutiv.

Bei der Kommunikation von Geschmacks erscheint es notwendig, zwei Anforderungen zu berücksichtigen. Zum einen müssen interaktiv ratifizierte Begründungen für Bewertungen angegeben werden. Dabei changieren diese Begründungen zwischen Bezugnahmen einerseits auf individuelle, subjektive Maßstäbe und Urteile sowie andererseits auf Geschmack als kollektiv gebildetes Phänomen, das auf im common ground verankerten Annahmen basiert (vgl. Kapitel 8). Zum anderen müssen Bewertungen möglichst kontextuell so platziert werden, dass sie eigene epistemische Autorität signalisieren und nicht einfach nur Zustimmung markieren. Da zweite Bewertungen die Chance haben, das Stimmungsbild der Gruppe kollek- 
tiv zu verschieben (vgl. Abschnitte 6.3 und 7.3.2), kann ein als geteilt hergestellter Geschmack durchaus subjektive Bewertungen überlagern.

Die Analysen zeigen auch, dass Expertise und damit die epistemische Autorität zur Evaluation zugeschrieben und bestätigt werden muss. Aus den Datenanalysen geht hervor, dass die Teilnehmenden in diesem Zuge einander anzeigen, kompetente Rezipient`innen zu sein. Kompetent bedeutet an dieser Stelle, dass sie einander demonstrieren, dass sie wissen, was sie tun, wenn sie Serien auswählen, anschauen und darüber sprechen. ,Serienprofis‘ werden im Rahmen dieser Studie daher als Personen verstanden, die sich als kompetente Serienrezipient ${ }^{\star}$ innen positionieren. So kann beispielsweise eine Teilnehmerin begründen, dass sie $\mathrm{SU}$ PERGIRL für das Bedürfnis nach Unterhaltung nutzt, und erhält dafür von der Gruppe Zustimmung (vgl. Abschnitt 8.1.3). Während ein anderer Teilnehmer vergleichbar in Bezug auf How I MET YOUR MOTHER argumentiert (Abschnitt 7.3.3), gerät er im Unterschied dazu in starken Begründungszwang. Seine präsentierte Kompetenz, bestimmte Serien für bestimmte Bedürfnisse auszuwählen, wird vor dem Hintergrund normativer Vorstellungen (hier: Trivialitätsdiskurs, vgl. Abschnitt 8.2.2) beurteilt und nicht interaktiv ratifiziert. Umgekehrt schreibt ein Teilnehmer drei anderen Gruppenmitgliedern Expertise bei der Frage nach türkischen Serien $\mathrm{zu}$, ohne dass diese eine entsprechende Kenntnis zuvor erläutert hätten (vgl. dazu Weiser-Zurmühlen 2020). Daran wird deutlich, dass Kompetenz ein interaktives Konstrukt ist und wechselseitig zugeschrieben werden muss.

Für die - erfolgreiche - Partizipation an Medienkommunikation muss also der eigene Geschmack in der Gemeinschaft verortet und dort als individuell und einzigartig kommuniziert, zugleich aber auch als geteilter Geschmack hergestellt werden. Das Gleiche gilt für Expertise: Es ist erforderlich, sich über das Besserbzw. Anders-Wissen einzelner Elemente zu distanzieren, sich aber gleichermaßen über einen geteilten common ground mit anderen zu vergemeinschaften. Exemplarisch wird das an der Positionierung eines Schülers als Serienrezipient, der außergewöhnliche Serien sehr gut kennt und schätzt deutlich, die interaktiv von den anderen Gruppenmitgliedern mehrfach gefestigt und bestätigt wird (vgl. Analysen in den Abschnitten 8.1.4 und 8.2.2). Diese Prozesse können mit Hilfe des erarbeiteten Modells analytisch erfasst und beschrieben werden. Dabei können sich die interaktiven Anforderungen zur Kommunikation des Geschmacks jederzeit dynamisch verändern, beispielsweise von emotional zu aggressiv (vgl. Abschnitt 9.1.1). Weiterhin können solche Positionierungen während eines Gesprächs im zeitlichen Verlauf kontinuierlich aufgegriffen werden und als interaktiv aufeinander bezogen verstanden werden.

Was zeichnet also Serienprofis als kompetente Serienrezipient*innen aus? Sie können die Klaviatur des Positionierens zur kontextuell passenden Vergemeinschaftung und Distinktion bedienen: Sie passen ihre bewertenden und epistemi- 
schen Äußerungen den kontextuellen Anforderungen entsprechend an und agieren im Sinne der jeweils relevanten interaktiven Ziele (Partizipation, Vergemeinschaftung und Distinktion) sowie der jeweils relevanten kommunikativen Aufgaben der Interaktionssituation. In diesem Zusammenhang betreiben sie den interaktiven Anforderungen entsprechend Wissensmanagement, d.h. sie können beispielsweise trailerähnliche, diskursive Formate produzieren. Sie geben den interaktiven Anforderungen entsprechende Begründungen für ihre Bewertungen an - das bedeutet auch, dass sie keine Begründungen angeben, wenn sie nicht erforderlich sind. Zum Positionieren als (un)wissende Unterstützer^innen oder Kritiker`innen nutzen sie mit Kategorisieren und Zuordnen, Kontrastieren und Vergleichen, Rekonstruieren und Inszenieren relevante Ressourcen. Insgesamt verorten sie ihren Geschmack auf einem Kontinuum zwischen außergewöhnlich und gemeinsam geteilt. Kurz gesagt: Serienprofis stellen Vergemeinschaftung und Distinktion den kontextuellen und interaktiven Anforderungen entsprechend her. Da Serien als Verstärker für Vergemeinschaftungs- und Distinktionsaktivitäten fungieren können, ist es möglich, sich ,über Umwege‘ zu positionieren: Indem Interagierende Serien positionieren, können sie zugleich implizit sich selbst und/oder andere positionieren.

\subsection{Zusammenfassung und Ausblick}

Im Rahmen der vorliegenden Studie wurde ein heuristisches Modell zur Analyse von Positionierungen im Kontext von Serienkommunikation entwickelt, mit dessen Hilfe es erstmals möglich ist, interaktive Vergemeinschaftungs- und Distinktionsaktivitäten in Form unterschiedlicher Konstellationen im Kontext von Medienkommunikation durch die Kombination mikro- und makroanalytischer Ansätze zu erfassen. Das Modell erlaubt es, die verschiedenen analytischen Dimensionen, die für diese Prozesse konstitutiv sind - den interaktiven Kontext, die epistemischen und evaluativen Positionierungspraktiken der Interagierenden sowie die relevant gesetzten Diskurse bezüglich des Gesprächsgegenstands, an denen sie sich orientieren - sowohl einzeln für sich zu betrachten als auch sie systematisch aufeinander zu beziehen und in ihrem Zusammenspiel zu interpretieren.

Für weitere Forschung zur Medienkommunikation erscheint es zielführend, Wissenskommunikation als konstitutiv für diese Art von Gesprächen zu betrachten, und die Frage, welche Interaktionsbeteiligten wie viel epistemischen Zugang zu dem medialen Gegenstand anzeigen, gezielt in Analysen einzubeziehen. Die Analyse von Bedeutungs- und Bewertungs(aus)handlungen erfordert es zudem, den Mikro- und Makrokontext systematisch aufeinander zu beziehen, denn in der Interaktion treffen subjektive Bedeutungszuweisungen und Werturteile sowie in 
Medienprodukte eingeschriebene Bedeutungen und diskursiv verankerte Bewertungen verdichtet aufeinander. Zugleich bleibt dabei aber auch herauszustellen, dass die Interaktion der Ort ist, an dem genau diese Prozesse empirisch fundiert beobachtet werden können. Die Frage „Was machen die Leute mit den Medien?“ (Katz und Foulkes 1962) ließe sich also für diesen Forschungsbereich modifizieren zu: „Was machen die Leute interaktiv mit den Medien?“

Vor diesem Hintergrund bietet es sich an, das Modell in anderen Kommunikationskontexten anzuwenden und zu modifizieren bzw. auszudifferenzieren. Die Analyseergebnisse dieser Studie legen zwar nahe, dass zwischen den Schulformen und Altersstufen kaum Unterschiede hinsichtlich der identifizierten Praktiken, ihrer Strukturstellen, Ausgestaltung und Funktionen bestehen. Hier würde sich aber eine größere systematische Untersuchung innerhalb der jeweiligen Schulformen und Altersgruppen anbieten. Weiterhin könnten auch Erwachsene als Proband`innen oder Positionierungen bezüglich anderer ästhetischer Erzeugnisse - Literatur, Kunst, Theater,... - als Objekt in den Blick genommen werden.

In der Einleitung wurde dargelegt, dass mittels Serien als Gesprächseinstieg bzw. Small Talk-Thema ad-hoc Vergemeinschaftung hergestellt werden kann. Die Analyseergebnisse zeigen, dass Vergemeinschaftung und Distinktion darüber hinaus als dynamische Prozesse insbesondere in Mehrparteien-Interaktionen zur Emergenz komplexer Konstellationen führen können. Sie legen zudem den Schluss nahe, dass es ebenso möglich ist, mit eher geringem epistemischen Zugang zu der besprochenen Serie an diesen Prozessen partizipieren zu können. Entscheidend dafür ist zum einen, bewertende Äußerungen kontextuell angemessen platzieren und adäquat in den Gesprächsverlauf integrieren zu können. Zum anderen ist es erforderlich, über ausreichend abstrakteres Wissen bezüglich Serien und Serialität zu verfügen. Dieses Ergebnis lässt sich auch auf andere Kontexte übertragen: Selbst wenn beispielsweise eine einzelne Theaterinszenierung oder literarisches Werk nicht bekannt ist, ist es möglich, an einem Gespräch darüber erfolgreich partizipieren zu können.

Da diese Studie mit Schüler*innen gearbeitet hat und die in der Einleitung zitierte Aussage von Anders und Staiger (2016), es handele sich bei ihnen - ohne, dass sie es selbst wüssten - um Serienprofis, für die vorliegende Studie in Richtung Interaktive Positionierung als Serienprofi präzisiert wurde, werden im Folgenden einige didaktische Anknüpfungsmöglichkeiten skizziert. Im Medienkompetenzrahmen NRW wird beispielsweise für den Kompetenzbereich ,Analysieren und reflektieren“ postuliert, eine „Bewegtbild- Lesekompetenz“4 sei für Schüler`innen

4 Vgl. https://medienkompetenzrahmen.nrw/unterrichtsmaterialien/detail/film-schule-nrw/ [zuletzt abgerufen am 06.04.2021]. 
im Digitalen Zeitalter unverzichtbar. Hier böte es sich an, mit den Schüler^innen Plausibilität der Handlungslogik und Figuren als ein Bewertungsmaßstab , den sie bereits intuitiv selbst anlegen, zu diskutieren und daran anzuknüpfen. Wird die hohe Relevanz von epistemischer Kommunikation bei Serien berücksichtigt, ließe sich gut mit vorhandenen Wissenselementen und -strukturen der Schüler`innen arbeiten; vergleichbar mit den Ausführungen von Schmidt und Winkler (2015), die dafür plädieren, im Literaturunterricht an informelles Filmwissen der Schüler^innen anzuknüpfen. So konnte gezeigt werden, dass die Teilnehmenden der vorliegenden Studie über prototypische Vorstellungen bezüglich diverser serieller Aspekte verfügen. Das ist insofern didaktisch relevant, als mit Prototypische Vorstellungen von Gattungen und Genres entwickeln ein zentraler Aspekt literarischen Lernens bezeichnet wird. Diese von Spinner (2006) formulierten Aspekte literarischen Lernens werden im deutschdidaktischen Diskurs mittlerweile auch für andere mediale Formate weiterentwickelt (vgl. die Beiträge in Ausgabe 2 der Zeitschrift Leseräume 2015), sodass Serien als Unterrichtsgegenstand hier sicher auch einen wertvollen Beitrag leisten können.

Somit ließen sich die vorhandenen Vorstellungen der Jugendlichen in den Unterricht integrieren und beispielsweise formale Merkmale von Serien in Abgrenzung zum Film erarbeiten oder die Funktionsweisen und typischen Konstellationen serieller Figuren diskutieren. Damit verbunden können auch die in dieser Studie herausgearbeiteten normativen Vorstellungen und Wertungen der Interagierenden, wie beispielsweise die impliziten Wertungen vermeintlich trivialer Formate, kritisch reflektiert werden. Letztlich bietet sich auch der für Serien konstitutive Aspekt des Spoilerns als Unterrichtsthema an. So kann besprochen werden, was einen Spoiler kennzeichnet und welche Konsequenzen sich ergeben, wenn jemand gespoilert wird. Daran könnte angeknüpft werden, indem die Schüler^innen selbst Trailer produzieren und kritisch beurteilen, ob diese Spoiler enthalten oder nicht. Für eine diesbezügliche Beurteilung ist sowohl eine gute Kenntnis des Werks an sich notwendig als auch darüber, wie Serien und Trailer funktionieren.

Insgesamt erscheint es zentral, Schüler*innen als kompetente Serienrezipient*innen ernstzunehmen, die Individualisierungstendenzen der Serienproduktion und -präsentation als Chancen zu begreifen und - innerhalb oder außerhalb der Schule - Gelegenheiten dafür zu schaffen, sich als Serienprofi zu positionieren. 